\title{
A weighted centroid localization algorithm based on RSSI adaptive value coupled with two norm improvements
}

\author{
Chao Cheng ${ }^{\text {a) }}$ Zhi-yang Jiang ${ }^{\text {b) }}$ \\ changchun university of technology, changchun 130012,china \\ a)jiangzhiyang921@163.com \\ b)1227064286@qq.com
}

\begin{abstract}
This paper proposes an improved localization algorithm based on signal strength (RSSI) adaptive value and two norm. In the localization process of the algorithm, the RSSI values as a direction vector, meet is qualitative, additivity is homogeneous and times, the RSSI values as a function with the concept of 'length'.In the actual positioning of the finite dimensional space, meet the Minkowski theorem and Cauchy convergence principle. The adaptive value and the second norm can effectively reduce the error of the weight and improve the positioning accuracy of the node. Through MATLAB simulation, the improved average positioning accuracy of this paper improved by $1.675 \mathrm{~m}$ compared with the traditional centroid positioning algorithm, and the optimization rate reached 52.8\%, which proves that the improved algorithm has some reference significance in positioning research.
\end{abstract}

Keywords: centroid, adaptive value, localization algorithm, signal strength,

\section{Introduction}

In the current trend of the Internet of things, the practical application of WSNs has been widely recognized, among which, positioning ${ }^{[1]}$ technology is a hot spot for its research content ${ }^{[2]}$. When estimating the position coordinates, it can be classified as the location mechanism based on ranging and non-ranging based on the use of distance. By contrast, the former can get smaller location accuracy, but high demand for hardware itself, in real life the main have maximum likelihood classification method, applied to the trilateral and triangulation method, etc. The node spacing can be in the process of $\mathrm{AOA}^{[3]}$, TOA $^{[4]}$, TDOA ${ }^{[5,6]}$, RSSI ranging technology to obtain ${ }^{[7]}$. If the coordinates of the unknown node are estimated by means of the location of the anchor node, the recombination of the region in the communication network structure or the number of routes, the latter algorithm is the latter. Although the error is relatively large, the dependence on hardware equipment is low and easy to operate. In this paper, the adaptive parameter combination 2 norm improved positioning method. According to the specific loss of signal intensity with letters punctuation, establish the corresponding adaptive reference value, and then corrected measured each RSSI values, and in view of the weighted formula optimization degree, is integrated into a 2 norm of thought to improve, the algorithm of measured their RSSI values as the direction of the vector, the weight of each anchor node coordinates and its inner product as one has the "length" function, can effectively reduce the loss of signal strength ability change of error, treat the positioning precision of the sensor nodes are largely improved.

\section{conventional positioning algorithm}

In the wireless network topology, the random distribution $\mathrm{I}$ is to be measured, and the location of $\mathrm{n}$ known nodes is not $\left(x_{1}, y_{1}\right),\left(x_{2}, y_{2}\right), \cdots,\left(x_{p}, y_{p}\right), \cdots,\left(x_{n}, y_{n}\right)$. Through the Zigbee module and other hardware devices, the signal strength loss value of the anchor node to the corresponding test node I is $r s s i_{i_{-}}, r s s i_{i_{-}}, \cdots, r s s i_{i_{-} P}, \cdots, r s s i_{i_{-} n}$. According to the loss of the signal in the transmission process, the influence degree of the reference point to the measuring point is reflected. The larger the loss, the smaller the effect. The proportion coefficient of each letter punctuation is defined as $1 /$ rssi , according to the following formula.

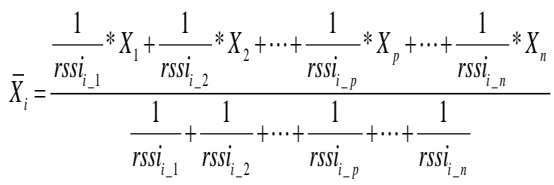


$\bar{Y}_{i}=\frac{\frac{1}{r s s i_{i_{-1}}} * Y_{1}+\frac{1}{r s s i_{i_{-} 2}} * Y_{2}+\cdots+\frac{1}{r s s i_{i-p}} * Y_{p}+\cdots+\frac{1}{r s s i_{i_{-n}}} * Y_{n}}{\frac{1}{r s s i_{i_{-} 1}}+\frac{1}{r s s i_{i_{-}}}+\cdots+\frac{1}{r s s i_{i_{-p}}}+\cdots+\frac{1}{r s s i_{i_{-n}}}}$

The estimated coordinate is $\left(\bar{X}_{i}, \bar{Y}_{i}\right)$ after the weighted optimization.

\section{This paper improves the weighted centroid positioning algorithm}

In traditional algorithms, the rssi corresponding to the letter punctuation coordinate is just a scalar. The first stage in this paper, the improved algorithm, the first through the establishment of adaptive value of the measured RSSI values, judge selected signal loss minimum value for rssi_min, this parameter is set to $R=r s s i \_m i n-1$, corresponding to each letter punctuation after the optimization of RSSI for $R S S I_{i_{-} 1}, R S S I_{i_{-} 2}, \cdots, R S S I_{i_{-} P}, \cdots, R S S I_{i_{-} n}$. Then, a parameter variable is set to ${ }^{M_{i}}=\sum_{p=1}^{n} r s i_{i_{-p}}$, and the weight between $\mathrm{k}$ and letter punctuation $\mathrm{p}$ is defined as $\frac{M_{k}}{\operatorname{RSI}_{k_{-} p}}$; In the second stage, the weighting factor is regarded as a function with the concept of "length". In the positioning process, a vector $\varphi_{k}(p)=\frac{M_{k}}{R S S I_{k_{-} p}}$ is introduced, and the estimated position coordinates of each of the nodes to be measured are.

$$
\begin{aligned}
& {\left[\begin{array}{cc}
X_{1}^{\prime} & Y_{1}^{\prime} \\
X_{2}^{\prime} & Y_{2}^{\prime} \\
\vdots & \vdots \\
X_{k}^{\prime} & Y_{k}^{\prime} \\
\vdots & \vdots \\
X_{i}^{\prime} & Y_{i}^{\prime}
\end{array}\right]=\left[\begin{array}{cccccc}
\varphi_{1}(1) & \varphi_{1}(2) & \cdots & \varphi_{1}(p) & \cdots & \varphi_{1}(n) \\
\varphi_{2}(1) & \varphi_{2}(2) & \cdots & \varphi_{2}(p) & \cdots & \varphi_{2}(n) \\
\vdots & \vdots & \vdots & \vdots & \vdots & \vdots \\
\varphi_{k}(1) & \varphi_{k}(2) & \cdots & \varphi_{k}(p) & \cdots & \varphi_{k}(n) \\
\vdots & \vdots & \vdots & \vdots & \vdots & \vdots \\
\varphi_{i}(1) & \varphi_{i}(2) & \cdots & \varphi_{i}(p) & \cdots & \varphi_{i}(n)
\end{array}\right]\left[\begin{array}{cc}
x_{1} & y_{1} \\
x_{2} & y_{2} \\
\vdots & \vdots \\
x_{p} & y_{p} \\
\vdots & \vdots \\
x_{n} & y_{n}
\end{array}\right]} \\
& =\left[\begin{array}{cc}
\varphi_{1}(1) * x_{1}+\varphi_{1}(2) * x_{2}+\cdots+\varphi_{1}(p) * x_{p}+\cdots+\varphi_{1}(n) * x_{i} & \varphi_{1}(1) * y_{1}+\varphi_{1}(2) * y_{2}+\cdots+\varphi_{1}(p) * y_{p}+\cdots+\varphi_{1}(n) * y_{n} \\
\varphi_{2}(1) * x_{1}+\varphi_{2}(2) * x_{2}+\cdots+\varphi_{2}(p) * x_{p}+\cdots+\varphi_{2}(n) * x_{i} & \varphi_{2}(1) * y_{1}+\varphi_{2}(2) * y_{2}+\cdots+\varphi_{2}(p) * y_{p}+\cdots+\varphi_{2}(n) * y_{n} \\
\vdots & \vdots \\
\varphi_{k}(1) * x_{1}+\varphi_{k}(2) * x_{2}+\cdots+\varphi_{k}(p) * x_{p}+\cdots+\varphi_{k}(n) * x_{i} & \varphi_{k}(1) * y_{1}+\varphi_{k}(2) * y_{2}+\cdots+\varphi_{k}(p) * y_{p}+\cdots+\varphi_{k}(n) * y_{n} \\
\vdots & \vdots \\
\varphi_{i}(1) * x_{1}+\varphi_{i}(2) * x_{2}+\cdots+\varphi_{i}(p) * x_{p}+\cdots+\varphi_{i}(n) * x_{i} & \varphi_{i}(1) * y_{1}+\varphi_{i}(2) * y_{2}+\cdots+\varphi_{i}(p) * y_{p}+\cdots+\varphi_{i}(n) * y_{n}
\end{array}\right]
\end{aligned}
$$

Because of compatibility, we can use the two norm to optimize the proportion of letter punctuation to the measurement point. According to the improved weighted centroid algorithm formula (3)(4), that is, $\left(X_{i}^{\prime \prime}, Y_{i}^{\prime \prime}\right)$ to be corrected by the algorithm in this paper.

$$
\begin{gathered}
X_{i}^{\prime \prime}=\frac{\left\|X_{i}^{\prime}\right\|_{2}}{\left\|\varphi_{i}(p)\right\|_{2}}=\frac{\left\|\sum_{p=1}^{n} \varphi_{i}(p) * x_{p}\right\|_{2}}{\left\|\varphi_{i}(p)\right\|_{2}}=\frac{\left(\left(\frac{M_{i}}{R S S I_{i-1}} * x_{1}\right)^{2}+\left(\frac{M_{i}}{R S S I_{i-2}} * x_{2}\right)^{2}+\cdots+\left(\frac{M_{i}}{R S S I_{i-p}} * x_{p}\right)^{2}+\cdots+\left(\frac{M_{i}}{R S S I_{i-n}} * x_{n}\right)^{2}\right)^{1 / 2}}{\left(\left(\frac{M_{i}}{R S S I_{i-1}}\right)^{2}+\left(\frac{M_{i}}{R S S I_{i-2}}\right)^{2}+\cdots+\left(\frac{M_{i}}{R S S I_{i-p}}\right)^{2}+\cdots+\left(\frac{M_{i}}{R S S I_{i-n}}\right)^{2}\right)^{1 / 2}} \\
Y_{i}^{\prime \prime}=\frac{\left\|Y_{i}^{\prime}\right\|_{2}}{\left\|\varphi_{i}(p)\right\|_{2}}=\frac{\left\|\sum_{p=1}^{n} \varphi_{i}(p) * y_{p}\right\|_{2}}{\left\|\varphi_{i}(p)\right\|_{2}}=\frac{\left(\left(\frac{M_{i}}{R S S I_{i-1}} * y_{1}\right)^{2}+\left(\frac{M_{i}}{R S S I_{i-2}} * y_{2}\right)^{2}+\cdots+\left(\frac{M_{i}}{R S S I_{i-p}} * y_{p}\right)^{2}+\cdots+\left(\frac{M_{i}}{R S S I_{i-n}} * y_{n}\right)^{2}\right)^{1 / 2}}{\left(\left(\frac{M_{i}}{R S S I_{i-1}}\right)^{2}+\left(\frac{M_{k}}{R S S I_{i-2}}\right)^{2}+\cdots+\left(\frac{M_{i}}{R S S I_{i-p}}\right)^{2}+\cdots+\left(\frac{M_{i}}{R S S I_{i-n}}\right)^{2}\right)^{1 / 2}}
\end{gathered}
$$




\section{Simulation results}

The PC used in this experiment is 4G memory and the Intel pentium processor of Windows7 system. The simulation platform is MATLAB, and the environment is simulated as the area of $10 \mathrm{~m} \mathrm{X} 10 \mathrm{~m}$, and the nodes are randomly scattered. The following is the simulation result comparing with the traditional .

Fig. 1 shows the comparison diagram of node estimation locations between different methods. It is very intuitive to see that the improved algorithm is higher in positioning accuracy than others.

Fig. 2 shows the error results obtained by the unknown nodes in the communication range in the algorithm of 5. This paper improved the average error is always at the bottom, after which the precision of the optimal estimation of the node under test point reached $0.99 \mathrm{~m}$, even more than the highest precision of the improved integral algorithm improved the $0.37 \mathrm{~m}$.

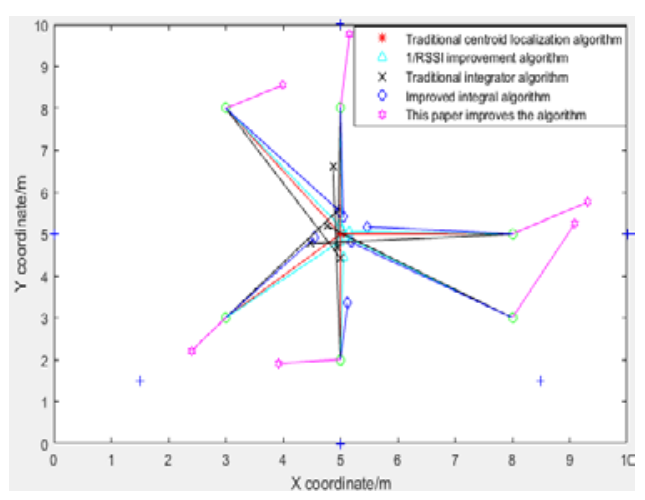

Fig. 1 the error of the estimated node is directly shown

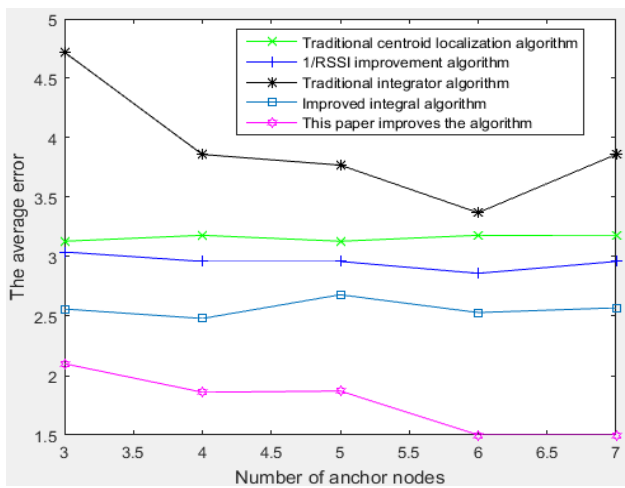

Fig. 3 the average error simulation results of

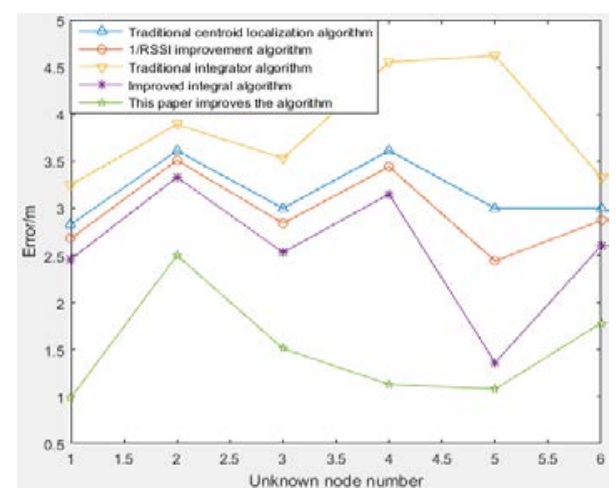

Fig. 2 error results of each of the measured points

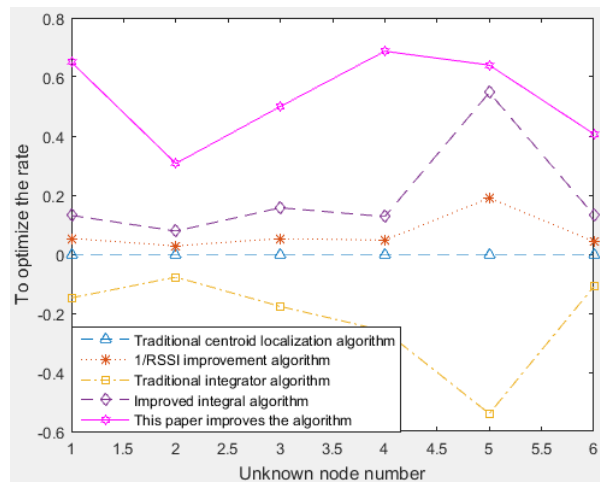

Fig. 4 optimization of each node

the change of anchor nodes

Fig. 3 shows the average error graph measured by the comparison method with the change of the target points. In this paper, the improved method in specific estimation results reflect the significant effect of optimization, compared with several other algorithms, The basic value and the norm of positioning mechanism more effectively reduces the signal strength in the process of transmission loss of nonlinear error, the improved algorithm with the increase of the number of anchor nodes, the error change quite gentle, in punctuation letter number for June and July, nodes can be measured by the position precision is $1.50 \mathrm{~m}$.

Fig. 4 can be found that the traditional centroid localization algorithm, this paper can achieve higher demands on the results, the node of the highest degree of optimization and even reached $68.7 \%$.

\section{Conclusion}

Aiming at the nonlinear error caused by the unstable loss of the wireless signal in the transmission process, an improved weighted centroid algorithm based on RSSI adaptive parameters is proposed. The 
algorithm in the first phase of the measured RSSI values are optimized, to redefine the communication within the scope of the influence degree of each anchor node coordinates, moreover in the second stage into the thought of two norm, the introduction of vector, the weighted factor and the letters punctuation as a vector has direction, by solving the norm has the function of the concept of "length", in which the dependent variable as the signal strength value and the known node coordinates, to optimize positioning results. In the simulation experiment, it also shows that the algorithm has a certain degree of feasibility in the evaluation index of positioning technology, and the results are more obvious. It has great reference value both in the novelty and the execution of the algorithm. Subsequent work intends to further study the channel factors of signal transmission.

\section{ACKNOWLEDGMENTS}

This work was financially supported by jilin provincial science and technology department fund.

About the author:

Chao Cheng, male, (1984-), PhD, lecturer, research direction for short - range wireless communication technology. E-mail: chengx415@163.com.

Zhi-yang Jiang (1993-), male, han nationality, master graduate, engaged in the research of wireless sensor network. E-mail: jiangzhiyang921@163.com.

\section{REFERENCES}

[1]Xu Xiao-long,Tang Yu,Wang Xin-heng,Zhang Yun.Variance-based fingerprint distance adjustment algorithm for indoor localization[J]. Journal of Systems Engineering and Electronics,2015,26-(6):1191-1201.

[2] Liu Rui-rui,WANG Yun-long,YIN Jie-xin ,Wang Ding,etal. Passive source localization using importance sampling based on TOA and FOA measurements $[\mathrm{J}]$. Frontiers of Information Technology \& Electronic Engineering,2017, 08(18):1167-1180.

[3] Naeimi Soroush,Chow Chee-onn,Ishii Hiro-shi.Directional multi-hop clustering routing protocol for wireless sensor networks [J] . International Journal of Ad Hoc and Ubiquitious Comp-uting,2013,14(2): 123-134.

[4] Niculescu D and Nath B.Ad hoc positioning system(APS) using AOA[C] . IEEE INFOCOM 2003:The Conference on Computer Communic-ations,San Francisco,2003:1734-1743.

[5] Radhika Nagpal,Howard Shrobe,Jonathan Bachrach.Organizing a global coordinate system form local information on an Ad hoc sensor network[C]. 2nd International Workshop on Information Processing in Sensor Networks (IPSN ‘03),palo Alto,2003:1-16.

[6] Ho K C.Bias reduction for an explicit solution of source localization using TDOA[J].IEEE Trans-actions on Signal Processing,2012,60(5):2101-2114.

[7]Cheng Chao, Qian Zhi-hong, Fu Cai-xin, Liu Xiao-hui. Genetic Optimization DV-Hop Loc-alization algorithm based on error distance weighting and jump segment algorithm [J]. Journal of electronics and information, 2015, 37 (10) : 2418-2423. 